\title{
Advent of a new sequencing era: long-read and on-site sequencing
}

\author{
Yutaka Suzuki ${ }^{1}$
}

Received: 30 September 2019 / Accepted: 2 October 2019 / Published online: 6 January 2020

(c) The Author(s), under exclusive licence to The Japan Society of Human Genetics 2019

Ten years have passed since the introduction of the socalled next-generation sequencers. The related technologies have revolutionized a wide variety of research fields, and a great diversity of biological research fields has begun to be explored. Particularly for medical applications, the sequencing analysis of cancers and other diseases has identified a large number of disease-causing mutations or variations in the human genome. Based on the initial mutation catalogue, "clinical sequencing"-which is the sequencing-based diagnosis for patients-is rapidly becoming a practical method in clinical laboratories in hospitals worldwide. Not only in humans but also in other organisms such as mice and other model organisms for basic sciences, plants for agricultural purposes, and microbes involved in environmental sciences and infectious diseases, multifaceted utilization of sequencing technologies has been initiated, as in genome-typing, gene expression, and epidemiological studies of given organisms.

Recently, a new type of sequencer has been developed. These collectively called "third- or fourth-generation sequencers" are "single molecular"-type sequencers, which are represented by the PacBio sequencer and Nanopore sequencer. Even though versatile applications have just started, already it has been envisioned that they will bring no less impact than the current next-generation sequencers. This new type of sequencer is still under development, having an obvious disadvantage in its sequencing fidelity. However, at the same time, it has substantial advantages over the previous sequencers. For example, this sequencer can read DNA more than $100 \mathrm{~kb}$ in length. Several applications have begun making use of these advantages. Indeed, the new sequencer has already revealed its potential in its applications for diseased genome sequencing in humans either by the de novo assembly approach or by the mapping-based approach.

Also noteworthy, a representative sequencer of the Nanopore type, MinION from Oxford Nanopore Technologies, is a disposable sequencer by which sequencing is possible without any pre-installation of expensive sequencers or any specific skills in biological experiments. By connecting MinION to a laptop PC, even an electrical supply is not required. This feature of MinION has opened a new opportunity for sequencing analysis in field areas of developing countries where high initial investment is not always affordable. Indeed, DNA sequencing has been performed in jungles, high mountains, Antarctica, and even in the Space Station. Information infrastructure, which accommodates the analysis of the produced data, is also being developed. Now these new types of sequencers are leveraging the potential power of sequencing truly worldwide, so that DNA sequencing technology is now bringing many new challenges for researchers throughout the world.

Here in this special issue is a series of review and original papers, so that the latest applications of these new types sequences can be collectively represented. This special issue also covers rapidly developing related bioinformatics tools and emerging related technologies. I sincerely hope the information described herein will be helpful for a wide variety of readers as they contemplate the new challenges in this post-next-generation sequencing era.
Yutaka Suzuki

ysuzuki@hgc.jp

1 Department of Computational Biology and Medical Sciences, The University of Tokyo, 5-1-5 Kashiwanoha, Kashiwa, Chiba 2778562, Japan 